\title{
Supplementary information: Determination of nanostructure of liposomes containing two model drugs by X-ray scattering from a synchrotron source
}

\author{
Alexandr Nasedkin, ${ }^{a}$ Jan Davidsson ${ }^{a}$ and Mont Kumpugdee-Vollrath ${ }^{b^{*}}$ \\ ${ }^{a}$ Department of Chemistry - Ångström Laboratory, Uppsala University, \\ Lagerhyddsvägen 1, S-75120 Uppsala, Sweden, and \\ ${ }^{b}$ Department of Pharmaceutical Engineering, Faculty of Mathematics-Physics- \\ Chemistry, Beuth Hochschule für Technik Berlin - University of Applied Sciences; \\ Luxemburger Str. 10, Berlin, 13353, Germany.E-mail:vollrath@beuth-hochschule.de
}

\section{Scattering model and fitting the experimental data}

The X-ray scattering model employed for data analysis has been developed and described in detail by Pabst et al. (2000). Modeled phospholipid electron density profile was represented by a combination of three Gaussian profiles: two for the two head-groups and one for the tail region (Fig. 2b). The equilibrium electron density baseline corresponds to electron density of the $\mathrm{CH}_{2}$ tail. In total nine parameters were variables in the fitting:

$\rho_{\mathrm{H}}-$ maximum density of the head group

$\rho_{\mathrm{C}}-$ minimum density of the chain group of atoms

$\sigma_{\mathrm{H}}$ - broadness of the head group Gaussian distribution

$\sigma_{\mathrm{C}}$ - broadness of the chain group Gaussian distribution

$z_{\mathrm{H}}$ - distance between the center of bilayer and the center of head group (also referred as head-to-midlayer distance)

$d$ - repetitive distance between bilayers

$N$ - number of bilayers in the wall

$\eta$ - Caillé parameter

$N_{\text {diff }}$ - diffusive scattering term

After the fitting performed, structural parameters could be derived from geometric relations to the bilayer profile. Main parameters of interest are:

headgroup size

hydrocarbon chain length,

bilayer thickness,

$$
\begin{aligned}
& d_{\mathrm{H}}=\sigma(\mathrm{FWHM})_{\mathrm{H}} \\
& d_{\mathrm{C}}=z_{\mathrm{H}}-\sigma(\mathrm{FWHM})_{\mathrm{H}} / 2 \\
& d_{\text {bilayer }}=2 *\left(z_{H}+\sigma(\mathrm{FWHM})_{\mathrm{H}} / 2\right)
\end{aligned}
$$


thickness of water layer, $\quad d_{\text {water }}=d-d_{\text {bilayer }}$

where $\sigma(\mathrm{FWHM})_{\mathrm{H}}$ is the full width at half maximum of the Gaussian representing the head-group, i.e. $2 \sigma_{\mathrm{H}} * 2 \ln 2$.

The lipid molecule surface area is given by:

$$
A=\frac{1}{\rho_{C H 2}\left(\frac{\rho_{H}}{\rho_{C}}-1\right)}\left(\frac{\rho_{H}}{\rho_{C}} \frac{n_{C}^{e}}{d_{C}}-\frac{n_{H}^{e}}{d_{H}}\right)
$$

where $n_{C}^{e}$ and $n_{H}^{e}$ are the number of electrons in the chain and the head-groups, respectively. The number of electrons was calculated based on the structure of the phospholipid head group and the average molecular weight of the phospholipids, assuming that the tails consist of only $\mathrm{CH}_{2}$-groups. The molecular weights of the phospholipid are $788 \mathrm{~g} / \mathrm{mol}$ for $85 \mathrm{G}$ lipids and $758 \mathrm{~g} / \mathrm{mol}$ for phospholipids $90 \mathrm{G}$ and $90 \mathrm{NG}$.

Finally, the scattering from lipid bilayer is determined by expression:

$$
I(q)=\frac{1}{q^{2}}\left(|F(q)|^{2} S(q)+N_{\text {diff }}|F(q)|\right)
$$

where $q$ is the scattering vector, $F(q)$ is the bilayer scattering form factor that is calculated in the simulation based on the six parameters defined above. $S(q)$ is the wall shape factor which depends on the number of layers in the wall and on the amplitude of fluctuations of monolayers caused by mechanical stress (described by the Caillé parameter $\eta$ ). An additional diffusive scattering term $N_{\text {diff }}$ is added in order to take care of internal wall defects and fluctuations.

The errors in determination of fitting parameters presented in Tables S1-S3 are the highest estimated. The error bar combines both experimental and modeling uncertainties. The biggest modeling uncertainty is introduced when assumed that all liposomes have the same number of bilayers in the wall. In practice the numbers of bilayers $N$ have some distribution. To count for it, $N$ was varied by unity after the best fit obtained and experimental curve was fitted again with fixed value of $N$. The other parameters determined from such fittings and the best fitting were averaged over all the experimental curves and the error was calculated from these data. Thus, the experimental error is lower then the uncertainty which might be introduced by using current model. 
a)
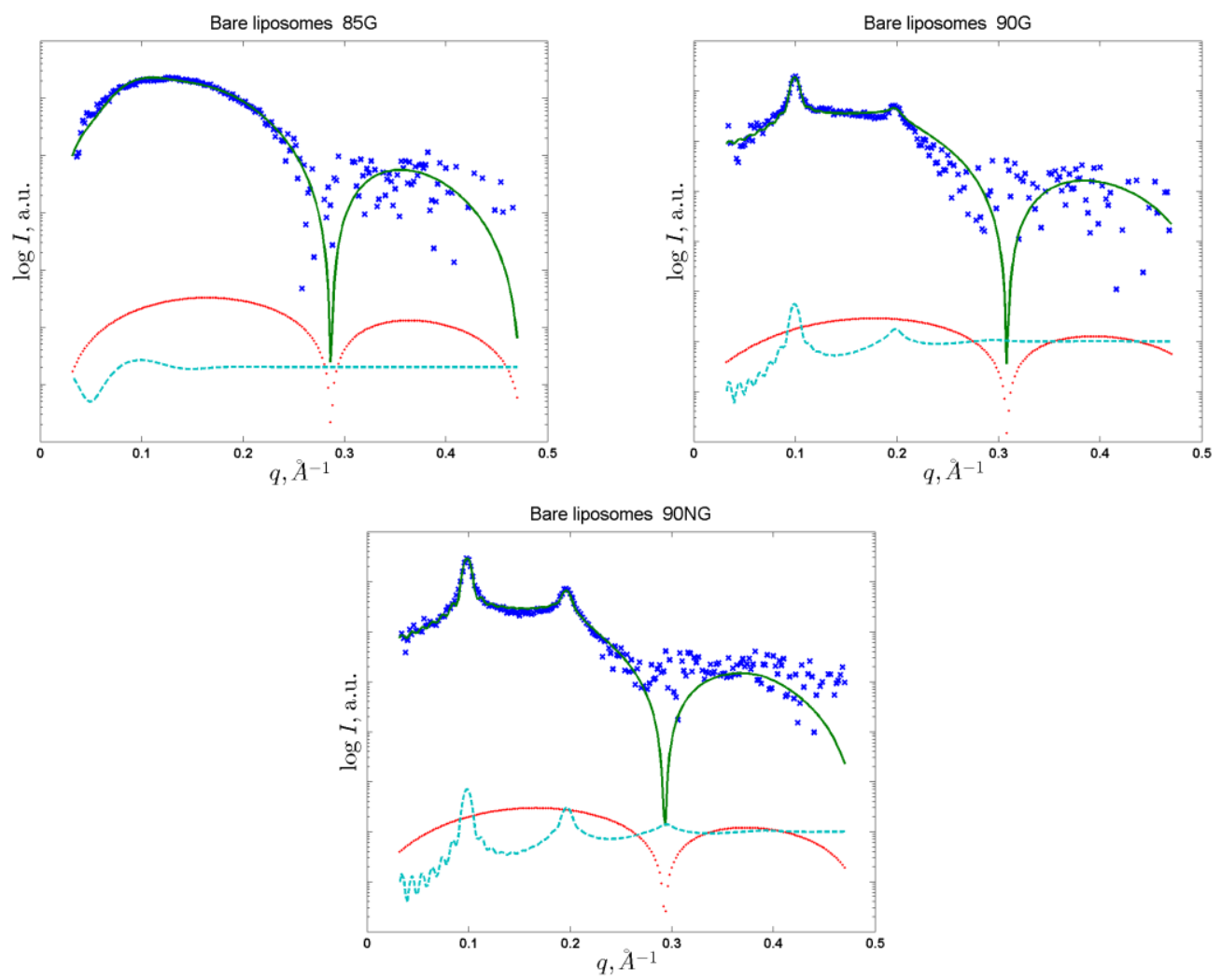

b)
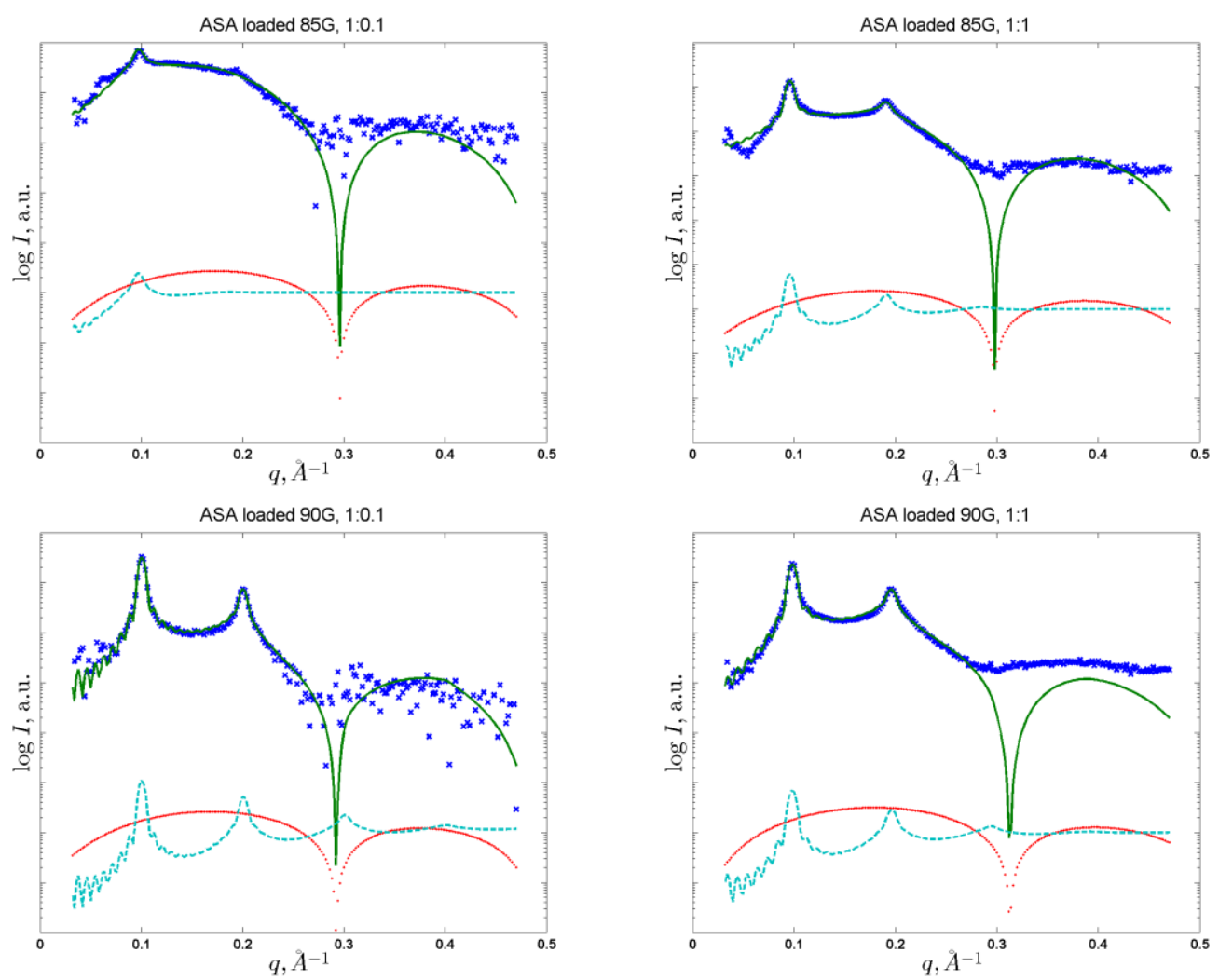

Page 3 of 5 

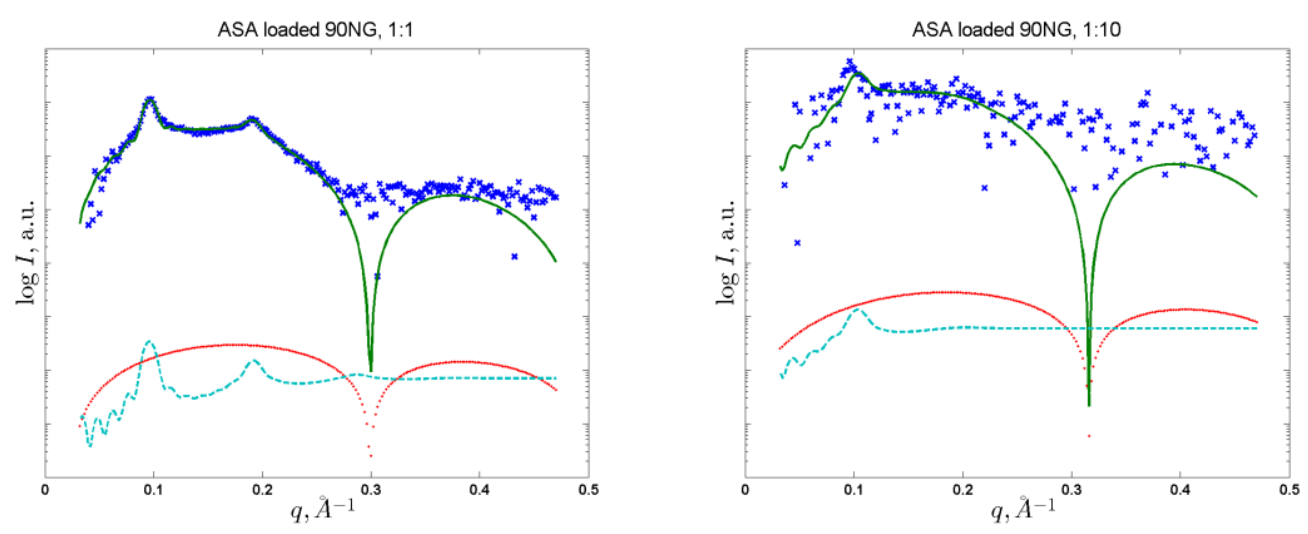

c)
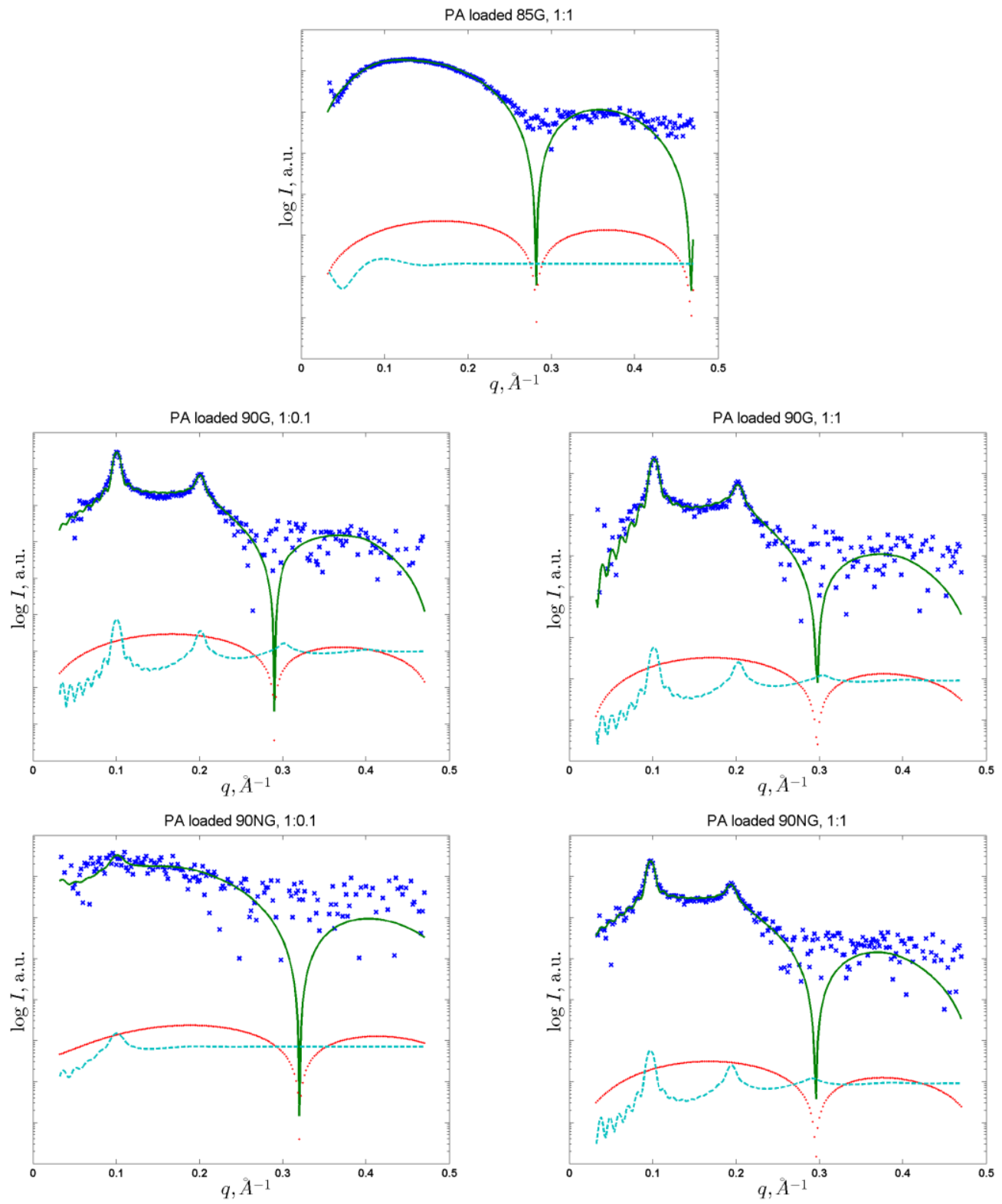

Figure S1 Examples of fitted model to the experimental scattering data: a) bare liposomes, b) acetylsalicylic acid loaded liposomes, c) paracetamol loaded liposomes. Blue crosses $(\mathrm{x})$ represent experimental data, green solid line (-) is fitted model, red dots (...) indicate scattering form factor and cyan dash line (--) indicates scattering shape factor. 
Table S1 Fitted parameters for different types of bare liposomes in water

\begin{tabular}{lccc}
\hline Phospholipid type & $85 \mathrm{G}$ & $90 \mathrm{G}$ & $90 \mathrm{NG}$ \\
\hline Number of analyzed curves & 7 & 5 & 7 \\
$\sigma_{\mathrm{H}}, \AA$ & $3.3 \pm 0.4$ & $3.0 \pm 0.3$ & $3.2 \pm 0.3$ \\
$\sigma_{\mathrm{C}}, \AA$ & $9.9 \pm 0.3$ & $10.3 \pm 0.5$ & $9.8 \pm 0.3$ \\
$\rho_{\mathrm{H}}, e / \AA^{3}$ & $0.11 \pm 0.01$ & $0.11 \pm 0.01$ & $0.10 \pm 0.01$ \\
$\rho_{\mathrm{C}}, e / \AA^{3}$ & $-0.08 \pm 0.01$ & $-0.08 \pm 0.01$ & $-0.08 \pm 0.01$ \\
$z_{\mathrm{H}}, \AA$ & $16.4 \pm 0.8$ & $16.6 \pm 0.6$ & $15.9 \pm 0.5$ \\
$d, \AA$ & $60.1 \pm 0.2$ & $63.4 \pm 0.3$ & $63.8 \pm 0.6$ \\
$N$ & $2 \pm 1$ & $9.6 \pm 2$ & $9.8 \pm 2$ \\
$C$ aillé parameter $\eta$ & $0.45 \pm 0.24$ & $0.47 \pm 0.29$ & $0.13 \pm 0.05$ \\
$N_{\text {diff }}$ & $4.0 \pm 0.8$ & $6.2 \pm 1.3$ & $3.1 \pm 0.7$ \\
\hline
\end{tabular}

Table S2 Fitted parameters for acetylsalicylic acid-loaded liposomes

\begin{tabular}{lcccccc}
\hline Phospholipid type & $85 \mathrm{G}$ & $85 \mathrm{G}$ & $90 \mathrm{G}$ & $90 \mathrm{G}$ & $90 \mathrm{NG}$ & $90 \mathrm{NG}$ \\
\hline Ratio of lipid to drug & $1: 0.1$ & $1: 1$ & $1: 0.1$ & $1: 1$ & $1: 1$ & $1: 10$ \\
Number of analyzed curves & 2 & 3 & 2 & 3 & 5 & 2 \\
$\sigma_{\mathrm{H}}, \AA$ & $2.4 \pm 0.5$ & $2.7 \pm 0.1$ & $3.1 \pm 0.1$ & $3.4 \pm 0.3$ & $2.9 \pm 0.1$ & $3.1 \pm 0.2$ \\
$\sigma_{\mathrm{C}}, \AA$ & $10.6 \pm 0.2$ & $11.0 \pm 0.4$ & $9.8 \pm 0.1$ & $9.7 \pm 0.2$ & $9.8 \pm 0.1$ & $9.6 \pm 0.1$ \\
$\rho_{\mathrm{H}}, e / \AA^{3}$ & $0.10 \pm 0.01$ & $0.13 \pm 0.01$ & $0.10 \pm 0.01$ & $0.12 \pm 0.01$ & $0.12 \pm 0.01$ & $0.12 \pm 0.01$ \\
$\rho_{\mathrm{C}}, e / \AA^{3}$ & $-0.05 \pm 0.01$ & $-0.06 \pm 0.01$ & $-0.07 \pm 0.01$ & $-0.09 \pm 0.01$ & $-0.07 \pm 0.01$ & $-0.07 \pm 0.01$ \\
$z_{\mathrm{H}}, \AA$ & $16.9 \pm 0.1$ & $15.8 \pm 0.2$ & $16.2 \pm 0.3$ & $15.1 \pm 0.1$ & $15.9 \pm 0.1$ & $15.4 \pm 0.3$ \\
$d, \AA$ & $64.0 \pm 0.2$ & $65.6 \pm 0.1$ & $62.6 \pm 0.1$ & $63.9 \pm 0.1$ & $65.3 \pm 0.1$ & $64.8 \pm 0.1$ \\
$N$ & $10 \pm 1$ & $10 \pm 1$ & $12 \pm 1$ & $10 \pm 1$ & $7 \pm 1$ & $5.5 \pm 1$ \\
Caillé parameter $\eta$ & $0.52 \pm 0.32$ & $0.20 \pm 0.03$ & $0.10 \pm 0.01$ & $0.15 \pm 0.02$ & $0.18 \pm 0.01$ & $0.32 \pm 0.02$ \\
$N_{\text {diff }}$ & $12.3 \pm 8.6$ & $4.6 \pm 0.3$ & $\leq 0.1$ & $0.98 \pm 0.32$ & $5.7 \pm 1.4$ & $\leq 0.01$ \\
\hline
\end{tabular}

Table S3 Fitted parameters for paracetamol-loaded liposomes

\begin{tabular}{lccccc}
\hline Phospholipid type & $85 \mathrm{G}$ & $90 \mathrm{G}$ & $90 \mathrm{G}$ & $90 \mathrm{NG}$ & $90 \mathrm{NG}$ \\
\hline Ratio of lipid to drug & $1: 1$ & $1: 0.1$ & $1: 1$ & $1: 0.1$ & $1: 1$ \\
Number of analyzed curves & 3 & 2 & 3 & 1 & 2 \\
$\sigma_{\mathrm{H}}, \AA$ & $2.8 \pm 0.3$ & $3.4 \pm 0.2$ & $3.6 \pm 0.2$ & $3.1 \pm 0.2$ & $3.5 \pm 0.1$ \\
$\sigma_{\mathrm{C}}, \AA$ & $10.0 \pm 0.1$ & $9.7 \pm 0.1$ & $9.7 \pm 0.1$ & $10.0 \pm 0.1$ & $9.6 \pm 0.1$ \\
$\rho_{\mathrm{H}}, e / \AA^{3}$ & $0.11 \pm 0.01$ & $0.11 \pm 0.01$ & $0.12 \pm 0.01$ & $0.11 \pm 0.01$ & $0.11 \pm 0.01$ \\
$\rho_{\mathrm{C}}, e / \AA^{3}$ & $-0.06 \pm 0.02$ & $-0.08 \pm 0.01$ & $-0.08 \pm 0.01$ & $-0.07 \pm 0.01$ & $-0.08 \pm 0.02$ \\
$z_{\mathrm{H}}, \AA$ & $16.7 \pm 0.2$ & $16.3 \pm 0.2$ & $16.2 \pm 0.3$ & $15.6 \pm 1.2$ & $16.2 \pm 0.2$ \\
$d, \AA$ & $60.2 \pm 0.3$ & $62.4 \pm 0.1$ & $61.9 \pm 0.1$ & $62.6 \pm 4.5$ & $64.5 \pm 0.1$ \\
$N$ & $2 \pm 1$ & $10 \pm 1$ & $9 \pm 1$ & $7 \pm 1$ & $9 \pm 1$ \\
Caillé parameter $\eta$ & $0.47 \pm 0.20$ & $0.11 \pm 0.03$ & $0.11 \pm 0.03$ & $0.70 \pm 0.23$ & $0.14 \pm 0.02$ \\
$N_{\text {diff }}$ & $9.9 \pm 3.8$ & $3.2 \pm 0.5$ & $1.5 \pm 0.3$ & $3.8 \pm 0.7$ & $3.9 \pm 0.45$ \\
\hline
\end{tabular}

